Linguistique, littérature, didactique

\title{
Les diseurs de vérité ou de l'éthique énonciative.
}

Parrèsiastes, messagers, whistleblowers, lanceurs d'alerte

Truth Tellers \& Enunciative Ethics. Parresiasts, Messengers, Whistleblowers

\section{Marie-Anne Paveau}

\section{(2) OpenEdition}

Journals

Édition électronique

URL : http://journals.openedition.org/pratiques/2267

DOI : $10.4000 /$ pratiques.2267

ISSN : 2425-2042

Éditeur

Centre de recherche sur les médiations (CREM)

Édition imprimée

Date de publication : 31 décembre 2014

Référence électronique

Marie-Anne Paveau, «Les diseurs de vérité ou de l'éthique énonciative. », Pratiques [En ligne],

163-164 | 2014, mis en ligne le 31 décembre 2014, consulté le 19 avril 2019. URL : http://

journals.openedition.org/pratiques/2267; DOI : 10.4000/pratiques.2267

Ce document a été généré automatiquement le 19 avril 2019

(c) Tous droits réservés 


\title{
Les diseurs de vérité ou de l'éthique énonciative.
}

\author{
Parrèsiastes, messagers, whistleblowers, lanceurs d'alerte \\ Truth Tellers \& Enunciative Ethics. Parresiasts, Messengers, Whistleblowers
}

Marie-Anne Paveau

\section{NOTE DE L'AUTEUR}

NDLA : Une partie de cet article a été publié une première fois en 2012, dans les Cahiers de recherche de l'École doctorale en Linguistique française, 6, numéro spécial Hommage à Camillo Marazza, coordonné par Mariagrazia Margarito et Enrica Galazzi, Milan, Lampi di Stampa, p. 197-212, sous le titre «Les diseurs de vérité ou de l'éthique énonciative ». Merci aux coordinatrices du numéro d'avoir généreusement accepté sa republication ici. Le titre a été augmenté, la partie sur les whistleblowers a été augmentée et approfondie, la section sur les lanceurs d'alerte a été ajoutée et les références, les dates et la bibliographie ont été complétées et actualisées.

\section{Introduction}

1 Dire la vérité, quoi de plus évident? Dans les représentations courantes qui constituent souvent la morale non questionnée de nos sociétés, la vérité nous semble valorisée, et même valorisante. Si l'on y regarde de plus près, on se rend compte que l'énoncé de la vérité a une valeur ambigüe: celui ${ }^{1}$ qui dit la vérité est souvent marginalisé, voire condamné.

Après avoir décrit rapidement la manière dont les philosophes et le sens commun posent le problème de la vérité, je présenterai trois figures de locuteurs de vérité, les « diseurs de vérité », le messager, le parrèsiaste et le whistleblower, à partir desquelles peut se poser la 
question de l'articulation entre éthique et linguistique, à travers l'énonciation et la profération.

\section{Dire la vérité : pratiques et représentations}

2 L'impératif de véracité se rencontre dans toutes les grandes cultures: dans les sagesses asiatiques, la véracité est une valeur fondamentale, et Les Entretiens de Confucius contiennent par exemple de nombreuses maximes qui soulignent le devoir de sincérité :

- Tseng Tzeu dit: «Je m'examine chaque jour sur trois choses: si, traitant une affaire pour un autre, je ne l'ai pas traitée sans loyauté ; si, dans mes relations avec mes amis, je n'ai pas manqué de sincérité; si je n'ai pas négligé de mettre en pratique les leçons que j'ai reçues » (2005 : I. 4).

- Le Maître dit: "Si un homme honorable manque de gravité, il ne sera pas respecté et sa connaissance ne sera pas solide. Qu'il mette au premier rang la loyauté et la sincérité; qu'il ne lie pas amitié avec des hommes qui ne lui ressemblent pas; s'il tombe dans un défaut, qu'il ait le courage de s'en corriger » (2005: I, 8).

3 La tradition chrétienne prône l'amour et le respect de la vérité en toute occasion et condamne le mensonge : c'est le discours tenu par saint Augustin dans le De mendacio, repris par Thomas d'Aquin pour lequel le mensonge est «mauvais». Pour M. de Montaigne, héritant des traditions antique et chrétienne dans les Essais, le mensonge est un « maudit vice» («Des menteurs »: I-IX), et s'oppose avec la méchanceté aux qualités de vérité et de générosité qui sont le propre des gens courageux («De la présomption »: II-XVII). Mais cette conception témoigne également d'une sociologie avant la lettre, l'auteur des Essais condamnant le mensonge également parce qu'il menace la cohésion sociale: «Notre intelligence se conduisant par la seule voie de la parole, celui qui la fausse, trahit la société publique » (« Du démentir » : II-XVIII).

Position qui se retrouve presque intacte dans la pragmatique moderne, le principe de sincérité de P. Grice étant paraphrasable par cette maxime ancienne. Les moralistes du $\mathrm{XVII}$ siècle ne cesseront de défendre la vérité et la sincérité, dans le cadre d'une conception de la parole participant de l'éducation morale: la préface des Caractères rappelle qu'« on ne doit parler, on ne doit écrire que pour l'instruction » (La Bruyère, 1976 : 118). Les philosophes des Lumières, qui défendent la vérité dans les usages des mots, font aussi l'éloge de certaines valeurs, comme la sincérité : pour Montesquieu (1995 : 78), elle est « la vertu qui fait l'honnête homme dans la vie privée et le héros dans le commerce des grands ".

Je ne vais pas détailler ce fil de la vérité et de la sincérité qui court dans l'ensemble de l'histoire de la pensée ${ }^{2}$, mais je dois m'arrêter cependant sur l'une des manifestations les plus connues, dans l'histoire de la philosophie, de cet attachement à la véracité, qui est la position d'E. Kant par rapport au mensonge. Dans la célèbre controverse qui l'oppose à $\mathrm{B}$. Constant en 1796 à propos de l'exemple classique du mensonge pour protéger un fugitif poursuivi par un assassin, publiée sous le titre Le droit de mentir (2003), il présente le devoir de vérité comme sacré dans un texte intitulé « La véracité est un devoir absolu et inconditionnel » :

«C'est donc un commandement sacré de la raison, un commandement qui n'admet pas de condition, et qu'aucun inconvénient ne saurait restreindre, que celui qui nous prescrit d'être véridiques (loyaux) dans toutes nos déclarations » (Kant, 2003 [1796] : 49). 
Pour B. Constant au contraire, « tout le monde n'a pas droit à la vérité » :

«Dire la vérité est un devoir. Qu'est-ce qu'un devoir? L'idée de devoir est inséparable de celle de droits : un devoir est ce qui, dans un être, correspond aux droits d'un autre. Là où il n'y a pas de droits, il n'y a pas de devoirs. Dire la vérité n'est donc un devoir qu'envers ceux qui ont droit à la vérité. Or nul homme n'a droit à la vérité qui nuit à autrui » (Constant, 2003 [1796] : 35).

5 Dans cette tradition, la vérité est donc une valeur morale et un principe universel. On trouve des traces de cette conception dans de nombreux discours, en particulier dans le discours politique, où la promesse de sincérité et l'engagement de vérité est un stéréotype discursif. Un exemple (presque) au hasard extrait d'un discours de vœux de N. Sarkozy en 2007 :

"J'ai voulu mettre chacun face à ses responsabilités. J'ai pris les miennes. J'ai pu commettre des erreurs. Mais depuis huit mois, je n'ai agi qu'avec le souci de défendre les intérêts de la France et pas un jour ne s'est passé où je ne me sois répété l'engagement que j'ai pris envers chacun de vous : “Je ne vous tromperai pas, je ne vous trahirai pas". Je vous dois la vérité, je vous la dirai toujours, je ne m'autoriserai aucune hypocrisie » (N. Sarkozy, Vœux aux Français, 31/12/07).

Le lexique de l'engagement et l'autocitation de la "maxime personnelle » du sujet illustrent bien la notion déontologique du devoir de véracité. En même temps, la politique est habitée par le mensonge, comme le montrent de nombreux travaux sur la question en science politique, des dissimulations de Nixon déclenchant le fameux «Watergate » à toutes les autres affaires de secrets, d'écoutes et de mensonges qui surgissent régulièrement dans la vie politique de tous les pays, l'élément -gate étant même devenu un outil néologique productif (Camillagate, Monicagate, Foreclosuregate, Irangate et tout récemment $D S K$-gate). La vie sociale elle aussi est ponctuée de "mensonges blancs ", selon une appellation issue de Thomas d'Aquin, qui arrondissent les angles et évitent les guerres, froides ou déclarées. Et il n'est pas si sûr que la vérité soit toujours considérée comme une chose bonne (à dire) et conforme à la morale.

\section{Le messager de vérité, une figure ambigüe}

7 Une plongée dans les dictionnaires des proverbes du monde contredit en effet à la fois l'idée d'un devoir universel de vérité, la nécessité naturelle de la vérité dans la langue et jusqu'au caractère vertueux de la vérité. Une bonne partie des proverbes, tous pays et langues confondus, qui mentionnent la vérité la présentent en effet comme un discours dangereux, à même de condamner son diseur à la marginalité, l'exil, voire la mort. Quelques exemples :

Trois sortes de gens disent la vérité : les sots, les enfants et les ivrognes

Une vieille erreur a plus d'amis qu'une nouvelle vérité

On frappe toujours le violoneux de la vérité avec son propre archet

Celui qui dit la vérité doit s'attendre à être expulsé de neuf villes

Qui dit tout haut la vérité risque de manquer d'abri

Celui qui dit toujours la vérité ne trouvera jamais un asile

Si tu dis la vérité, point ne pèches, mais que de maux tu suscites !

Avec la vérité qui nous accompagne, on va partout, même en prison!

Celui qui dit la vérité doit avoir son cheval sellé

8 Être un messager de vérité n'est donc guère confortable, et cela dans presque toutes les cultures. L'inconfort se transforme en danger si la nouvelle apportée est à la fois vraie et 
funeste. Il existe de nombreux exemples philosophiques et littéraires de cette figure négative du messager. L'adage d'Érasme «Legatus non caeditur, neque violatur» (Adages: 4.7.20) insiste sur cette ambigüité périlleuse du statut du diseur de vérité. Cette conception de la parole insupportable du messager survient deux fois dans L'Antigone de Sophocle, de la bouche même du gardien au moment où il annonce que Polynice a reçu une sépulture («Les mauvaises nouvelles sont fatales à qui les apporte», v. 276), et plus tard de la part de Créon quand le messager vient lui dire la mort d'Eurydice (« Messager, courrier d'atroces tourments, que viens-tu m'apporter?», v. 1290). L'adage est repris par W. Shakespeare dans Henri IV et Le Roi Lear, et l'expression s'est déclinée en différentes variantes. Ne tirez pas sur le messager fournit en effet une sorte de modèle discursif qui varie ses cibles : ne tirez pas sur le pianiste, sur le shérif, sur le trader, et même sur la coccinelle asiatique (rencontré sur un blog de jardinage) ou sur les Lions indomptables (trouvé dans un article sur la célèbre équipe de football camerounaise).

Le messager inaudible est presque une figure de la sagesse morale et politique, comme le montre la fameuse maxime de N. de Chamfort, dans un chapitre sur la France, l'esclavage et la liberté : « En France, on laisse en repos ceux qui mettent le feu, et on persécute ceux qui sonnent le tocsin " (Chamfort, 1923 [1795]: VIII, D, 171). On n'aime pas celui qui apporte une mauvaise nouvelle, parce qu'il annonce des contenus malheureux, certes, mais surtout parce qu'il dérange l'ordre social et trouble le confortable déni qui fait tenir les sociétés.

B. Stanford a intitulé l'un de ses livres Don't Shoot the Messenger: How Our Growing Hatred of the Media Threatens Free Speech for All of Us: il y montre que la haine des messagers de mauvaises nouvelles que sont les médias remet gravement en cause la liberté d'expression dans l'ensemble de la société. À partir du moment où l'on «tire sur le messager ", alors la vérité est bâillonnée et ne sont alors plus formulées que les «bonnes » nouvelles ou les informations acceptables parce que consensuelles.

La vérité n'est pas toujours bonne à dire, dit le proverbe. La figure du messager, « homme à abattre », est l'une des incarnations de cette sagesse ambigüe. Il en existe une autre, issue de la philosophie grecque, le parrèsiaste.

\section{Le parrèsiaste ou le courage de la vérité}

Dans son dernier cours au Collège de France donné en 1984, édité sous le titre Le courage de la vérité (2009), M. Foucault développe longuement une figure antique du diseur de vérité, le parrèsiaste, celui qui pratique la parrêsia. Elle «consiste à dire, sans dissimulation ni réserve ni clause de style ni ornement rhétorique qui pourrait la chiffrer et la masquer, la vérité. Le "tout-dire" est à ce moment-là : dire la vérité sans rien en cacher, sans la cacher par quoi que ce soit » (Foucault, 2009: 11). Traduisible selon lui par «franc-parler", la parrêsia est un dire-vrai qui constitue une vertu essentiellement politique. Il existe également un sens apostolique et spirituel de la parrêsia, trait fondamental de la parole du prophète. La notion apparait assez souvent dans le Nouveau Testament où elle est plutôt traduite dans le vocabulaire de la confiance, de l'assurance et de la parole ouverte. Cette manière de "tout dire" recèle cependant l'ambigüité fondamentale attachée à la notion même de vérité : la parrêsia peut en effet devenir une menace pour la démocratie en y introduisant des différences de légitimité, et elle peut aussi franchir les limites psychiques de l'acceptable en se muant en franchise perverse. 
M. Foucault (2009: 12) précise dans Le courage de la vérité que dire le vrai n'est pas suffisant pour qu'il y ait parrêsia : «Il faut que le sujet, en disant cette vérité qu'il marque comme étant son opinion, sa pensée, sa croyance, prenne un certain risque, risque qui concerne la relation même qu'il a avec celui auquel il s'adresse ». Et c'est de ce risque que vient, en termes éthiques, la valeur de ce dire vrai : « D'où ce nouveau trait de la parrêsia : elle implique une certaine forme de courage, courage dont la forme minimale consiste en ceci que le parrèsiaste risque de défaire, de dénouer cette relation à l'autre qui a rendu possible précisément son discours » (Foucault, 2009 : 13). M. Foucault peut alors donner de la parrêsia une définition synthétique, qui présente la notion dans le cadre d'un contrat de parole :

«La parrêsia est donc, en deux mots, le courage de la vérité chez celui qui parle et prend le risque de dire, en dépit de tout, toute la vérité qu'il pense, mais c'est aussi le courage de l'interlocuteur qui accepte de recevoir comme vraie la vérité blessante qu'il entend » (Foucault, 2009 : 14).

La notion de parrêsia et la figure du parrèsiaste, dont Démosthène est un prototype, est présente chez Sénèque, Plutarque, Gallien. M. Foucault (2009 : 37) insiste sur la vertu de courage qu'il présente comme véritablement structurelle à la parrêsia, courage qu'il associe également à la noblesse : « Un homme par conséquent qui parle pour des motifs nobles, et qui, pour ces motifs nobles, s'oppose à la volonté de tous, celui-là, dit Socrate, s'expose à la mort ». Mais c'est sur la philosophie cynique qu'il s'arrête longuement pour illustrer cette posture de parole aux dimensions éthique et politique. Il mentionne en particulier une description du philosophe cynique par Epictète, qui propose une figure bien intéressante pour notre série, celle de l'éclaireur :

«Epictète explique que le rôle du cynique, c'est d'exercer la fonction d'espion, d'éclaireur. Et il emploie le mot kataskopos, qui a un sens précis dans le vocabulaire militaire : ce sont des gens qu'on envoie un peu en avant de l'armée pour regarder aussi discrètement que possible ce que fait l'ennemi. C'est cette métaphore qu'Epictète utilise ici, puisqu'il dit que le cynique est envoyé comme éclaireur en avant, au delà du front de l'humanité, pour déterminer ce qui, dans les choses du monde, peut être favorable à l'homme ou peut lui être hostile » (Foucault, 2009 : 154).

Après cette marche en avant toujours solitaire, l'éclaireur, explique M. Foucault (2009: 155), revient parmi les hommes pour « annoncer la vérité, annoncer les choses vraies sans [...] se laisser paralyser par la crainte ». Cette pratique du dire vrai est articulée à un mode de vie, dont la célèbre figure de Diogène est l'illustration : un mode de vie qui a éliminé «toutes les conventions inutiles » et «toutes les opinions superflues", une philosophie qui réduit la vie à elle-même : «Je n'ai ni femme, ni enfant ni palais de gouverneur, mais la terre seule et le ciel et un vieux manteau. Et qu'est-ce qui me manque ? Ne suis-je pas sans chagrin et sans crainte, ne suis-je pas libre?» (parole du philosophe cynique dans les Entretiens d'Epictète).

Parrêsia est également un mot important dans le lexique de la Bible, qui reçoit des traductions un peu différentes de "franc-parler» ou "dire vrai ». Les équivalents appartiennent plutôt au champ de la confiance, qui, quand elle se fait parole publique, correspond à la franchise ou la liberté de parole. La traduction du mot n'est pas stable dans la Bible, et il est donc difficile de repérer le mot dans le texte en français. N. Adeline (2006) décrit cette situation lexicale :

«Dans le Nouveau Testament les attestations de ces deux mots sont nombreuses: parrêsia et parrêsiazomai se rencontrent une quarantaine de fois et nous noterons que la répartition de ces attestations est irrégulière. Aucun emploi chez Matthieu. 
Un seul emploi chez Marc. Mais des emplois dans l'Évangile selon Jean et le reste dans les Actes et les Epîtres. Rien d'étonnant à ce qu'on rencontre souvent, dans les textes centraux de la proclamation apostolique, un mot qui exprime l'assurance et la force de la conviction. »

Il cite ensuite plusieurs passages où apparait, derrière ses traductions, le mot de parrêsia. Par exemple, dans l'Évangile selon saint Jean, Jésus répond à des contradicteurs avec "hardiesse » lors de la fête des Tentes et impressionne les habitants de Jérusalem qui n'osent cependant, par crainte, «parler ouvertement » de ce diseur de vérité : « Personne ne parlait librement de Jésus » (Jean 7,13). Il s'adresse alors à la foule, et se décrit luimême en parrèsiaste: "Quelques habitants de Jérusalem disaient: "N'est-ce pas celui qu'ils cherchent à tuer? Le voilà qui parle librement, et les Juifs ne lui disent rien" " (Jean 7, 26).

Dans les Actes des apôtres, cette qualité de parole attribuée à Jésus touche aussi Pierre et Jean, qui comparaissent devant le Sanhédrin, accusés de diffuser les enseignements de Jésus. Les chefs des Juifs, les anciens et les scribes sont étonnés devant « l'assurance » des apôtres: "En voyant l'assurance de Pierre et de Jean, ils étaient étonnés, car ils se rendaient compte que c'étaient des gens du peuple sans instruction. Ils reconnaissaient en eux ceux qui étaient avec Jésus. Mais comme ils voyaient debout auprès d'eux l'homme guéri, ils n'avaient rien à répliquer (Actes 4, 13-14).

$\mathrm{N}$. Adeline conclut en présentant « un des passages les plus riches du Nouveau Testament sur les valeurs de la conviction, de l'assurance, de la parrêsia ». Ce sont les dernières paroles de Jésus à ses disciples :

« Je vous ai dit tout cela en discours figurés. L'heure vient où je ne vous tiendrai plus de discours figurés, mais où je vous annoncerai ouvertement ["en toute clarté" dans une autre version] ce qui concerne le Père. En ce jour-là vous demanderez en mon nom, et je ne vous dis pas que c'est moi qui demanderai au Père pour vous; en effet le Père lui-même est votre ami, parce que vous, vous avez été mes amis et vous avez acquis la conviction que, moi, je suis sorti de Dieu. Je suis sorti du Père et je suis venu dans le monde ; maintenant je quitte le monde et je vais vers le Père » (Jean 16, 25-28).

Et les disciples reprennent les paroles de Jésus : «Maintenant tu parles ouvertement et tu ne tiens plus des discours figurés. Maintenant nous savons que tu sais tout et que tu n'as besoin que personne t'interroge ; c'est pourquoi nous croyons que tu es sorti de Dieu " (Jean 16, 29).

On voit donc, à partir de ces deux traditions, la philosophie cynique et la naissance du christianisme, que la parrêsia appartient à un type de vérité hétérodoxe, qui va à l'encontre des pratiques langagières habituelles et reconnues d'une société ou d'un groupe. Le diseur de vérité n'est pas un simple locuteur; c'est un prophète, audacieux et combatif, marginal et condamné (parfois à mort), qui assume toutes les ambigüités du discours de vérité quand il prend une dimension politique.

Le parrèsiaste est donc un locuteur muni de cette vertu de courage qui est pour Aristote une vertu politique, celle de l'homme libre, et qui sera d'ailleurs pour Thomas d'Aquin la première des vertus, celle qui permet les autres. En même temps, il est aussi porteur d'une menace politique, la démocratie se trouvant considérablement fragilisée par le dire vrai, qui introduit une hiérarchie de valeur dans les différents discours. M. Foucault (2009: 148) formule très clairement cette ambigüité de la vérité publiquement et donc politiquement dite. D'un certain côté, la parrêsia empêche la folie totalitaire qui repose sur la soumission silencieuse des citoyens : 


\begin{abstract}
« À partir du moment où on n'a pas la parrêsia on est [...] obligé de supporter la sottise des maîtres. Et rien de plus dur que d'être fou avec les fous, d'être sot avec les sots. Cette mention du fait que, sans parrêsia, on est en quelque sorte soumis à la folie des maîtres, cela veut dire quoi et montre quoi ? Eh bien, cela montre que la parrêsia a pour fonction justement de pouvoir limiter le pouvoir des maîtres. Quand il y a de la parrêsia, et que le maître est là - le maître qui est fou et qui veut imposer sa folie, que fait le parrèsiaste, que fait celui qui pratique la parrêsia? Eh bien justement, il se lève, il se dresse, il prend la parole, il dit la vérité. Et contre la sottise, contre la folie, contre l'aveuglement du maître, il va dire le vrai, et par conséquent limiter par là la folie du maître. A partir du moment où il n'y a pas de parrêsia, alors les hommes, les citoyens, tout le monde est voué à cette folie du maître ».
\end{abstract}

De l'autre, la parrêsia est rendue impossible par la conservation de la démocratie: les sociétés et institutions démocratiques, explique M. Foucault, ne peuvent accueillir ni même supporter le discours de la vérité sur ce mode de la franchise, car il faudrait alors soutenir l'idée d'un "partage éthique » ou « différenciation éthique ». Cela conduirait à distinguer les citoyens selon leur degré de proximité avec la vérité, donc de faire la différence « entre ceux qui sont bons et ceux qui sont mauvais, entre les meilleurs et les pires ", ce qui contrevient à l'idée même de démocratie comme " champ politique défini par l'indifférence entre les sujets parlants » (Foucault, 2009 : 44). On verra plus bas à quel point cette analyse de M. Foucault est juste : les sociétés ne supportent pas, dans tous les sens du terme, que les vérités, surtout celles qui ne sont pas bonnes à dire, soient dites, et elles ont en ce sens un lourd programme de travail, à la fois social, discursif et politique, à accomplir pour l'amélioration éthique de la circulation de la parole.

\title{
4. Le whistleblower ou le tragique de la vérité
}

En 1987, S. Langton réalise The Whistle Blower, un film tiré d'un roman de J. Hale qui porte le même titre. Michael Caine y interprète Frank Jones, vétéran de la Seconde Guerre mondiale porteur d'une haute idée de la Grande-Bretagne, qui enquête sur la mort mystérieuse de son fils Bob, employé comme spécialiste de russe par les services secrets britanniques. Le slogan du film est: "Dans le monde secret des dissimulations gouvernementales, le seul homme qui ose dire la vérité survivra-t-il ? ». L'enquête se révèle difficile et dangereuse, Frank Jones affrontant le maillage épais du silence, du mensonge et de la corruption, qui recouvre la vérité, inaudible en pleine guerre froide, du meurtre de son fils. Il devient donc cet homme « qui parle pour des motifs nobles, et qui, pour ces motifs nobles s'oppose à la volonté de tous» dont parle Socrate. Comme le roman, le film est largement occupé par des réflexions sur le dévoilement impossible de la vérité, la puissance de la raison d'État et la culture de la dissimulation des agents de services secrets.

\subsection{Le sifflet de la révélation}

Le whistleblower, c'est celui qui donne un coup de sifflet (to blow a whistle), c'est-à-dire qui révèle un dysfonctionnement grave qui a lieu dans une organisation. Au sens propre, le terme désigne le policier qui signale une infraction ou un crime par un coup de sifflet destiné à la fois à appeler ses collègues et à alerter la population d'un danger. Métaphoriquement il désigne un individu qui pratique le "whistleblowing ", c'est-à-dire qui révèle «des pratiques illégales, immorales ou illégitimes couvertes par ses 
employeurs, à des personnes ou des organisations susceptibles d'intenter une action ", selon la définition proposée par deux juristes australiens (Brown, Latimer, 2008 : 768). La plupart des whistleblowers sont des "internal whistleblowers ", c'est-à-dire qu'ils révèlent des dysfonctionnements dans leur propre société ou organisation, la révélation concernant une organisation extérieure à l'agent étant plutôt rare. Il existe quelques figures célèbres de whistleblowers, régulièrement citées et constituées en exemples: Sherron Watkins, qui a révélé le scandale Enron en 2001, Joe Darby, à l'origine des révélations sur les maltraitances et les pratiques de torture à la prison d'Abu Graïb en 2004 ou Jeffrey Wigand, qui révèle dans les années 1990 comment la firme de tabac qui l'emploie ment sur les effets de la nicotine. Un film a été réalisé à partir de cette histoire, The Insider (un film de Michael Mann sorti en 1999 et interprété par Russell Crowe), et d'autres whistleblowers sont mis en scène au cinéma, la plupart du temps dans des blockbusters soutenus par des acteurs prestigieux, comme la célèbre Erin Brokovich, qui donne son nom à un film de Steven Soderbergh en 2000, où le personnage est interprété par Julia Roberts. Le "film de whistleblower» est désormais un véritable sous-genre cinématographique. En 2009, l'affaire Wikileaks a mis sur le devant de la scène un whistleblower géant: le site est en effet entièrement dédié au whistleblowing, dans la plus pure tradition américaine de la révélation des procédures cachées, du démasquage des mensonges d'État et de la mise au jour de la vérité, avec en prime la figure romanesque de son fondateur, Julian Assange, et l'utilisation inédite jusqu'à présent du support de diffusion du web.

En 2013, on apprend qu'Edward Snowden, 29 ans, employé chez un sous-traitant de la National Security Agency (NSA) depuis quatre ans, est le fameux whistleblower qui a révélé au public via le quotidien britannique The Guardian l'impressionnant programme de surveillance électronique PRISM des services secrets américains. Les États-Unis sont en effet en mesure d'avoir accès aux données détenues par Google, Facebook, YouTube, Microsoft, Yahoo !, Skype, AOL et Apple. Ce qui m'intéresse chez Edward Snowden, comme chez Julian Assange mentionné plus haut, ou Chelsea Manning ${ }^{3}$ et les « Catholic Whistleblowers » dont je parle plus bas, est évidemment de l'ordre du discours et concerne trois points : les modalités discursives des informations diffusées, le discours que les whistleblowers tiennent sur leur propre discours et leurs actes, et enfin le discours produit sur eux par les autres, en particulier par les instances mises en cause, entreprises ou gouvernements.

\subsection{Modalités discursives du whistleblowing}

Edward Snowden est informaticien, et ce sont les modalités de discours de sa profession, comme de son époque, qu'il utilise : il enregistre des fichiers sur quatre ordinateurs portables, et en envoie une partie à Glenn Greenwald, journaliste au Guardian (en fait c'est un circuit beaucoup plus complexe dont on trouvera les intéressants détails dans le livre de Glenn Greenwald lui-même, paru en 2014). Parmi eux, un fichier Powerpoint destiné aux employés de la NSA; on est donc dans de l'écrit numérique, transmis à distance, et ayant valeur de preuve, au nom de la crédibilité accordée au document écrit dans nos sociétés. Le whistleblower ne passera à l'oral qu'un peu plus tard, en accordant une interview à Glenn Greenwald, mais pour expliquer ses actes et décrire sa situation, et non pour le whistleblowing, qui reste exclusivement écrit et documentaire : c'est à l'écrit que l'on siffle. Chelsea Manning avait pour sa part emprunté un circuit discursif numérique préconstruit et destiné à la transmission d'information compromettantes : Wikileaks et sa 
page de "submissions", comportant un lien vers une drop box sécurisée. Comme Edward Snowden, son discours passe également par l'écrit documentaire puisqu'elle a transmis 150000 notes diplomatiques. Mais elle a également envoyé une vidéo classifiée, pour laquelle l'implicite de la preuve documentaire demeure : l'image fixe ou mobile élevée au statut de document est toujours plus crédible que le témoignage oral.

Les Catholic Whistleblowers (Goodstein, 2013) sont un peu particuliers. D'abord, il s'agit d'un groupe (ils sont douze, des prêtres et des religieuses), ce qui est parfaitement inédit dans l'histoire du whistleblowing, qui ne comptait jusqu'à présent que des voix solitaires. Ensuite, ce sont des femmes et hommes de livres plus que d'écrans, de parole orale plus que d'écrit numérique, même s'ils ont un site internet (http:// www.catholicwhistleblowers.com/). Ils émettent donc leurs « whistles » selon la littératie qui est la leur : ils écrivent des lettres (ils en ont écrit une au pape François en avril 2013) et se réunissent. Leur groupe a d'abord été un groupe de parole destiné à les soutenir euxmêmes dans leurs expériences de discours publics, et c'est progressivement qu'il s'est transformé en lieu de pratique du whistleblowing. Désormais, ils organisent des conférences et leur mode d'action est donc public, ce qui est sans doute rendu possible par le fait qu'ils constituent un groupe (pour le moment, le pape François n'a pas répondu à leur lettre).

\subsection{Le discours sur soi du whistleblower : le narcissisme moralisé}

Glenn Greenwald remarque que parmi tous les whistleblowers connus, aucun n'a jamais vendu d'informations pourtant très avantageusement monnayables. Au contraire, tous ont pris des risques importants, et presque tous se sont considérablement appauvris, voire pire (les travaux sur les whistleblowers mentionnent tous les issues fréquemment dramatique du whistleblowing: pertes d'emploi, divorces, addictions, et, parfois, suicides (voir par exemple Alford, 2001; 2004, Bowden, 2005, Faunce, 2004). Il décrit ainsi leur système moral: "They did not act with any self-interest in mind. The opposite is true: they undertook great personal risk and sacrifice for one overarching reason: to make their fellow citizens aware of what their government is doing in the dark. Their objective is to educate, to democratize, to create accountability for those in power " (The Guardian, 07/06/13). Edward Snowden, quant à lui, explique son geste de la manière suivante : «I don't want to live in a society that does these sort of things... I do not want to live in a world where everything I do and say is recorded. That is not something I am willing to support or live under " (The Guardian, 09/06/13, interview d'E. Snowden).

Dans les réponses qu'il donne aux questions d'internautes le 17 juin, constituant de passionnants échanges qui éclairent tant sur la personnalité de Snowden que sur les attitudes du gouvernement américain devant les paroles «non autorisées» de ses employés, et offrant aux analystes du discours une intéressante possibilité de corpus, il emploie les termes truth, transparency et lie, qui sont des termes du vocabulaire moral. Il y a donc de la morale dans le whistleblowing, même si cette interprétation n'est jamais vraiment explicite dans les discours médiatiques, y compris chez les défenseurs des whistleblowers eux-mêmes.

Dans ses travaux de philosophie morale sur la figure du whistleblower, C. F. Alford appelle «narcissisme moralisé » (narcissism moralized) ce rapport que le whistleblower entretient au discours de vérité. Dans Whistleblowers: Broken Lives and Organizational Power, publié en 2001, il explique que le narcissisme peut constituer « une source profonde et puissante de 
moralité, menant certains Narcisses à sacrifier leurs biens humains pour une idée noble " (Alford, $2001: 78$; ma traduction). Il y a donc chez les whistleblowers une pratique intense de la vertu discursive, en particulier en ce qui concerne l'ajustement de la parole à la vérité $\mathrm{du}$ monde ${ }^{4}$. Ils sont donc des agents vertueux, trop vertueux, et souvent malgré eux, ce que révèle la tournure doublement négative commune à plusieurs d'entre eux selon les enquêtes de C. F. Alford: "Je ne pouvais pas ne pas le faire». "Ne pas ne pas », « impossible de ne pas » : c'est là que se niche le narcissisme, dans la conservation d'une image de soi acceptable et aimable.

Chelsea Manning tient un discours analogue. Dans le témoignage qu'elle a rédigé en février $2013^{5}$, elle exprime à plusieurs reprises ce désir d'ajustement à la réalité que d'autres peuvent nommer "vérité »: «I felt this sense of relief by [WikiLeaks] having [the information]. I felt I had accomplished something that allowed me to have a clear conscience based upon what I had seen and what I had read about and knew were happening in both Iraq and Afghanistan everyday» (Manning, 2013, en ligne). Et, comme les autres, et comme Wikileaks également, qui utilise souvent le mot sur son site, il mentionne la notion de vérité : «I always want to figure out the truth. Unlike other analysts in my section [or other sections], I was not satisfied with just scratching the surface and producing canned or cookie-cutter assessments. I wanted to know why something was the way it was, and what we could do to correct or mitigate the situation » (Manning, 2013, en ligne).

Les Catholic Whistleblowers quant à eux sont évidemment placés dans une sorte de vertu au carré puisque le whistleblowing est, dans une vision idéale du clergé tout du moins, une part de leur métier : lutter contre le "mal », le "péché ", la souffrance infligée à autrui. Et les textes de leur site sont sans surprise saturés de vocabulaire moral. Mais ils ont également un souci d'image, un souci "narcissique »: "They say that their motivation is to make the church better and safer, and to show the world that there are good priests and nuns in the church ", peut-on lire sur leur page d'accueil.

\subsection{Un traitre au cheval sellé}

22 Ce n'est pas du tout la représentation qui se construit ailleurs, et évidemment dans les organismes, institutions ou groupes attaqués par le sifflet vertueux des diseurs de vérité. De nombreux observateurs décrivent une véritable méthode de diabolisation de la part du gouvernement américain, pays où la tradition du whistleblowing est fortement ancrée (mais elle l'est aussi dans le monde anglophone en général) :

"Ever since the Nixon administration broke into the office of Daniel Ellsberg's psychoanalyst's office, the tactic of the US government has been to attack and demonize whistleblowers as a means of distracting attention from their own exposed wrongdoing and destroying the credibility of the messenger so that everyone tunes out the message" (Greenwald, The Guardian, 07/06/13).

Les témoignages sont nombreux qui présentent le combat des whistleblowers comme des parcours du combattant pas toujours victorieux, et on peut lire sur ce point l'intéressant témoignage de Thomas Drake, ancien cadre supérieur de la NSA qui a dénoncé en 2010 le programme de modernisation de son agence, et dont le cas constitue désormais une référence en matière de whistleblowing (Drake, 2011). L'accusation de trahison est la plus fréquente, aggravée en environnement militaire par celle de contact avec l'ennemi (même s'il s'agit d'un contexte de paix). Cependant il me semble que la situation évolue, très certainement grâce au web, qui permet certaines désincarcérations : une pétition a été ouverte rapidement pour défendre Edward Snowden ( $\mathrm{A}$ White House petition to 
pardon Edward Snowden »), j'ai mentionné plus haut le site de soutien à Chelsea Manning et signalé que pour la première fois, des whistleblowers avaient formé un groupe, ce qui est une modification structurelle importante.

Les travaux de C. F. Alford, mais aussi de P. Bowden, A. J. Brown et P. Latimer, T. A. Faunce ou V.D. Lachman mentionnés en bibliographie soulignent le paradoxe du whistleblower: considéré comme un héros d'un côté, car il a travaillé pour le bien de la société et parfois pour la sauvegarde de la vie, il est, dans la pratique, isolé et mis à l'écart comme un facteur de trouble dans l'organisation sociale. Les représailles sont fréquentes, et les destins parfois tragiques. Le whistleblower incarne le paradoxe du statut de la vérité ellemême dans toutes les sociétés, de l'Antiquité à nos jours, paradoxe que montre bien M. Foucault (2009) dans Le courage de la vérité : la vérité comme idéal éthique, mais évitée dans la pratique car facteur de rupture, de violence, de perturbation. Le sifflet vertueux du whistleblower sonne encore comme une dérangeante crécelle aux oreilles du plus grand nombre. «Si tu aimes la vérité, tiens ton cheval sellé » est un proverbe que l'on trouve quasiment dans toutes les langues et toutes les cultures, sous des formes variées. C'est un sage conseil qu'a suivi Edward Snowden, qui a d'abord galopé jusqu'à Hong Kong, et qu'un Pégase moderne a transporté jusqu'en Russie.

Toutes les enquêtes montrent en effet que les whistleblowers qui diffusent au grand jour de mauvaises pratiques à grande échelle ou particulièrement dangereuses, sont la plupart du temps persécutés et courent de ce fait un véritable danger. La persécution des whistleblowers est devenue une question importante dans plusieurs pays: bien qu'ils soient en principe protégés des représailles de leurs employeurs par la loi, dans les faits de nombreuses affaires ont montré que les whistleblowers étaient sanctionnés par divers moyens, rétrogradation, retenues sur salaires, suspension, "mise au placard", harcèlement, et même maltraitance psychique grave.

C'est la raison pour laquelle plusieurs pays, en particulier l'Australie et les États-Unis, particulièrement en avance sur ce point, ont mis en place un arsenal juridique de protection. Aux États-Unis, des mesures de protection ont été prise depuis le False Claims Act qui a légitimé le whistleblowing en 1863, relayé par le Lloyd La Follette Act en 1912, jusqu'au récent Whistleblower Protection Act de 2007. Un " National Whistleblower Center " a été crée en 1988. En Australie, des lois de protection des whistleblowers ont été discutées et adoptées dans tous les états dans les années 1990 et 2000. Mais comme le signalent A. J. Brown et P. Latimer (2008: 768), «il est important que les lois sur les whistleblowers promeuvent une culture dans laquelle les révélations honnêtes soient respectées, valorisées et même récompensées ». La vertu du whistleblower dépend en effet de son environnement : les démocraties sont sans doute plus aptes à accepter le whistleblowing, puisqu'elles soutiennent en principe la transparence, la liberté d'expression et la responsabilité, que les régimes dictatoriaux ou autoritaires.

Certains auteurs travaillent à réduire voire éliminer cet inajustement, en particulier dans le domaine de l'éthique médicale. Le juriste australien T. A. Faunce, spécialisé dans le droit médical et l'éthique médicale, estime que le whistleblowing en milieu médical ( healthcare whistleblowing) souffre encore du paradoxe qui fait du whistleblower un " paria ", alors que toutes les études montrent que les whistleblowers sont très généralement sincères dans leur désir d'appliquer les vertus fondamentales et les principes de l'éthique médicale (Faunce, 2004). Pour lui, ce paradoxe est dû au fait qu'il n'existe pas, dans les théories comme dans les enseignements d'éthique médicale, de fondement éthique de ce comportement. Sa collègue américaine $\mathrm{V}$. Lachman, spécialiste de bioéthique à l'université Drexel de Philadelphie, est également très favorable à l'intégration d'une 
l'éthique du whistleblowing dans la formation médicale, de manière à ce que le whistleblowing en milieu médical finisse, un jour, idéalement, par disparaitre (Lachman, 2008).

La figure du whistleblower est donc une figure d'agent moral, que son discours soit qualifié de vertueux ou au contraire rejeté par la société comme inajusté aux valeurs de l'environnement. Comme le parrèsiaste, il est porteur d'un discours marqué par le courage de la vérité, et comme le parrèsiaste il court le risque d'être mis au ban de la société, car il publie des vérités insupportables.

\section{La figure française du lanceur d'alerte}

Le whistleblower, figure courante dans la culture anglophone, dans la réalité des pratiques et dans les discours, est au centre d'un important domaine de recherche scientifique. Les whistleblowers sont en effet des figures abondamment observées et théorisées aux ÉtatsUnis, au Canada, en Grande-Bretagne et en Australie. Il existe de nombreux travaux sur le whistleblowing en droit (une question essentielle étant sa protection juridique), en science politique, en sociologie, en éthique médicale, en psychologie, etc. Cette figure n'est pas traitée en France, où des sociologues autour de F. Chateauraynaud ont proposé celle du «lanceur d'alerte », très différente, en particulier sur la plan moral : si le whistleblower constitue une figure à fort coefficient d'éthicité, et travaillée comme telle par les chercheurs, la figure du lanceur d'alerte a été construite en dehors de la problématique morale, et, à l'origine, dans le contexte des risques naturels. Mais dans les faits, il existe parfois des confusions entre les deux notions.

La figure du lanceur d'alerte constitue elle aussi une postérité du parrèsiaste. Son intérêt, par rapport à la figure du whistleblower inscrite dans la perspective morale à la fois par les agents et les chercheurs, réside en ce que cette notion a été construite en dehors de la question morale, au sein d'une épistémologie sociologique française qui a parmi ses caractéristiques de maintenir l'axiologie, en particulier morale, à l'écart de ses théories et méthodes. Or, le lanceur d'alerte, comme avatar de la sentinelle cynique ou de la Cassandre antique, me semble cependant marqué par cette dimension morale de l'inajustement à l'environnement du dicible.

La notion de lanceur d'alerte a été élaborée vers 1995 en France par les sociologues F. Chateauraynaud et D. Torny pour nommer les « sombres précurseurs » (1999) que sont les savants qui signalent un risque futur. Contrairement aux whisteblowers dont les actions concernent surtout le monde de la finance, de la sécurité et de l'entreprise, les lanceurs d'alerte signalent surtout des risques environnementaux et évoluent plutôt dans les milieux scientifiques. En 2008, F. Chateauraynaud (2008: 1) en donne la définition suivante :

«La notion de lanceur d'alerte désigne toute entité, personne, groupe, institution, qui assume cette fonction d'alerte et qui cherche à faire reconnaître, souvent contre l'avis dominant, l'importance d'un danger ou d'un risque. [...] Pour naturelle que soit cette fonction d'alerte, elle se heurte, comme la plupart des actes orientés vers un public ou une institution, aux rapports de forces et de légitimités qui caractérisent, à une époque donnée, une formation sociale. La place des alertes et de ceux qui les lancent dépend ainsi de la configuration politique et des ressorts cognitifs communément disponibles, ainsi que des tensions ou des conflits qui les traversent. " 
liste des différents facteurs pouvant caractériser la fonction du lanceur d'alerte, politiques, cognitifs, sociaux, n'intègre pas la dimension morale. F. Chateauraynaud compare un peu plus loin les lanceurs d'alerte aux «guetteurs » ou «sentinelles » des sociétés animales, rejoignant en cela le lexique de $\mathrm{M}$. Foucault à propos des parrèsiastes. Il précise que la catégorie est élaborée dans un contexte scientifique précis, celui de travaux sur «la trajectoire des signes de danger », ce qui donne au lanceur d'alerte un rôle d'anticipateur que n'a pas le whistleblower, qui révèle au contraire des pratiques passées ou en train de se s'accomplir:

«A l'opposé du modèle du whistleblower, le lanceur d'alerte décrit celui (personne, groupe, institution) qui détecte des prodromes, des signes précurseurs sans avoir nécessairement d'interprétation ou de cadre prédéfini pour les qualifier. À l'idée du coup de sifflet, de l'arrêt d'un processus déclenché par un acteur qui a le pouvoir et l'autorité pour le faire, on a préféré celle d'une trajectoire ou même de la carrière d'un problème qui ne devient public que graduellement » (Chateauraynaud, 2008: 5).

comme les whistleblowers, les lanceurs d'alerte sont en général mal reçus, parce qu'ils brisent les équilibres discursifs, et ils sont souvent identifiés aux "prophètes de malheur ». Des chercheurs comme Pierre Meneton à l'Institut national de la santé et de la recherche médicale (Inserm), que ses travaux sur le sel opposent aux producteurs de sel, ou André Cicolella qui est le premier à dénoncer les dangers des éthers de glycol, ou encore, plus anciennement, Jean-François Viel qui souligne le nombre élevé de leucémies à La Hague, tous entrent dans cette catégorie de « lanceur d'alerte ». Tous ont eu, comme les whistleblowers, à affronter des pressions, des marginalistaions, des représailles, voire des procès. Mais $\mathrm{F}$. Chateauraynaud insiste beaucoup sur la différence entre les deux figures (il parle également parfois de "personnage») et souhaite éviter que le terme lanceur d'alerte n'apparaisse que comme une traduction de whistleblower. C'est que, explique-t-il, "la construction de la figure de l'alerte a été opérée en détachant analytiquement le moment de l'alerte et celui de la dénonciation ou de la critique, et en montrant que tout signal d'alerte engage une multiplicité de logiques d'action et de jugement comme la vigilance, la controverse, la polémique, la normalisation, l'accusation ou le scandale » (Chateauraynaud, $2009: 2$ ).

Cette distinction alerte/critique revient selon moi à la distinction fait/valeur, le phénomène socio-sémiotique de la transmission du signal étant présenté comme dissociable de son interprétation dans l'environnement. La tradition française, en sociologie tout du moins (mais la remarque vaut pour nombre de disciplines des sciences humaines et sociales), conserve soigneusement l'idée d'une objectivité de la science en objectivisant son objet lui-même, et en discriminant soigneusement des niveaux de réalité et d'interprétation: «Du point de vue de la protection de la parole publique de lanceurs d'alerte, l'essentiel est de pouvoir séparer la dénonciation et l'alerte, souligne le sociologue. Or dans les faits, [...] il est très difficile d'éviter que les deux régimes ne s'entremêlent » (Chateauraynaud, 2009: 7). L'idée d'un entremêlement des régimes, pour affaiblir la séparation radicale entre fait et valeur, la maintient cependant, et reste une visée souhaitable. Or, si l'on admet avec H. Putnam (2004 [2002]) « l'effondrement » de la distinction faits/valeurs, et si l'on adopte une vision plus globale du phénomène, qui ne permet pas de "démêler " les régimes, alors cette figure du lanceur d'alerte, comme toutes les figures et comme toutes les manifestations humaines, loin de pouvoir être décrite du seul point de vue de la circulation des signes de danger, doit être regardée aussi et en même temps sur le plan de la valeur, les deux points de vue étant indissociables. La 
parole du lanceur d'alerte est en effet inscrite dans une axiologie négative, imprégnée de morale: ce n'est pas une "bonne parole", mais plutôt un discours issu d'une «mauvaise » langue qui menace un ordre, économique et financier, mais également cet ordre du discours dont parlait M. Foucault et qui nous interdit de "tout dire». Cette imprégnation ne me semble pas séparable de l'analyse en terme de transmission de signes de danger, elle en est un composant. Il faut reconnaitre que l'interprétation éthique n'est pas totalement absente des analyses de F. Chateauraynaud, mais indirecte et centrée sur un moment du processus : « Il y a dans la trajectoire de toute alerte qui dérange au moins un moment critique, une phase dénonciatoire ou polémique, parfois même une bouffée paranoïaque, nécessaire au changement de régime» (Chateauraynaud, 2009: 3). Circonscrire la dimension axiologique à une «phase» de parole, l'associer d'emblée à la catégorie de la dénonciation, catégorie éthique qui caractérise à priori la parole comme négative et malveillante, ou la décrire par rapport à une " paranoïa " des récepteurs, ce sont me semble-t-il des manières de ne pas poser la question morale. L'indicibilité de la parole du lanceur d'alerte reste partiellement inexpliquée, et les « protocoles de prise en charge des alertes", que F. Chateauraynaud (2009: 7) souhaite enrichir de "modalités d'apprentissage, doublés d'une forme de didactique des régimes de prise de parole », me semblent incomplets.

\section{Conclusion}

Les diseurs de vérité posent une question fondamentale à l'analyse du discours, à la philosophie morale, mais aussi à la société tout entière : la place de la parole dissensuelle, hétérodoxe ou inaudible dans le concert des discours sociaux, et le sort réservé à celui qui sort du silence courtois ou de la silenciation violente. Les figures antiques du messager et du parrèsiaste, et celles plus contemporaines du whistleblower et du lanceur d'alerte, sont des figures d'énonciateur. En linguistique de l'énonciation et en analyse du discours, l'énonciateur est rarement l'objet du travail des description et de la théorisation, laissées sans doute à la psychologie ou l'anthropologie, les disciplines du langage restant largement logocentrées. Mais les diseurs, parleurs, proféreurs et autres producteurs de parole constituent pourtant des agents essentiels de toute vie sociale et de toute circulation de la parole, parce qu'ils s'expriment à partir d'une compétence langagière, d'une culture de la parole, d'une mémoire discursive et d'une table des valeurs morales qui imprègnent toutes leurs moindres discours.

\section{Bibliographie}

ADELINE, N. (2006) : « PARRÊSIA, l'assurance » (en ligne : http://biblique.blogspirit.com/ archive/2006/12/09/parresia-esperance.html, consulté le 30/03/15). 
ALFORD, C. F. (2001) : Whistleblowers: Broken Lives and Organizational Power, Ithaca, Cornell University Press.

- (2007) : «Whistleblower Narratives : The Experience of Choiceless Choice », Social Research, 74-1, p. 223-248 (en ligne : http://findarticles.com/p/articles/mi_m2267/is_1_74/ai_n19094732/, consulté le 30/03/15).

BOWDEN, P. (2005) : "A Comparative Analysis of Whistleblower Protection ", Australian Association for Professional and Applied Ethics, $12^{\text {th }}$ Annual Conference, 28-30 septembre, Adelaide.

BROWN, A. J. \& LATIMER, P. (2008) : «Whistleblower Laws : International Best Practice », UNSW Law Journal, 31-3, p. 766-794.

CHAMFORT, N. de (1923 [1795]) : Maximes et pensées, Paris, Éd. Grès.

CHATEAURAynAUD, F. (2008) : «Les lanceurs d'alerte dans l'espace politique. Réflexions sur la trajectoire d'une cause collective ", texte pour la journée Lanceurs d'alerte et système d'expertise : vers une législation exemplaire en 2008 ? (Paris, Sénat, 27 mars 2008) (en ligne : http:// gspr.ehess.free.fr/docs/FC/doc/doc-FC-2008-Lanceurs.pdf, consulté le 30/03/15). - (2009) : «Les lanceurs d'alerte et la loi », Experts, 83, p. 44-47.

CHATEAURAYNAUD, F. \& TORNY, D. (1999) : Les Sombres précurseurs. Une Sociologie pragmatique de l'alerte et du risque, Paris, Éd. de l'EHESS.

CONFUCIUS (2005) : Les Entretiens, Paris, Gallimard.

CONSTANT, B. \& KANT, E. (2003 [1796]) : Le droit de mentir, Paris, Éd. Mille et Une Nuits.

ARRIEN (2000) : Manuel d'Épictète [Epictète, Entretiens], trad., int. et prés. par P. Hadot, Paris, Éd. Le Livre de poche.

DRAKE, T. (2011) : «Is This the Country We Want to Keep ? ", Sam Adams Award Acceptance Speech, The Real News [site] (en ligne : http://therealnews.com/t2/index.php? option=com_content\&task=view\&id=31\&Itemid=74\&jumival=7755, consulté le 30/03/15).

ÉRASME, 2011, Les Adages, traduction (latin et grec) et édition dirigées par J.-C. Saladin, Paris, Éd. Les Belles Lettres.

FAUNCE, T. A. (2004) : « Developing and Teaching the Virtue-Ethics Foundations of Healthcare Whistle Blowing ", Monash Bioethics Review, 23-4, p. 41-55.

FOUCAULT, M. (2009) : Le courage de la vérité. Le gouvernement de soi et des autres II. Cours au collège de France 1984, éd. par F. Gros, Paris, Gallimard/Éd. Le Seuil.

Goodstein, L. (2013) : « Church Whistle-Blowers Join Forces on Abuse », The New York Times [site], 20 mai (en ligne : http://www.nytimes.com/2013/05/21/us/catholic-church-whistle-blowersjoin-forces-on-abuse.html?_r=4\&, consulté le 30/03/15)

GREENWALD, G. June 7 (2013) : " On Whistleblowers and Government Threats of Investigation », The Guardian [site], 7 juin (en ligne : http://www.theguardian.com/commentisfree/2013/jun/07/ whistleblowers-and-leak-investigations, consulté le 30/03/15).

- (2014) : Nulle part où se cacher, trad. Johan-Frederik Hel Guedj, Paris, J.-C. Lattès.

LA BRUYÈRE, J. de (1976 [1688]) : Les caractères ou les mœurs de ce siècle, Paris, Éd. Le Livre de poche.

LACHMAN, V. D., 2008, « Whistleblowers : Troublemakers or Virtuous Nurses ? ", MEDSURG Nursing, 17-2, p. 126-134.

FUlLER, N. (2013) : « In her Own Words », Free Chelsea Manning [site], 7 mars (en ligne : http:// www.chelseamanning.org/learn-more/in-his-own-words, consulté le 30/03/15). 
MANNING C. (2013): « Bradley Manning's Statement taking Responsibility for Releasing Documents to WikiLeaks ", Free Chelsea Manning [site], 28 février (en ligne : http://www.chelseamanning.org/ news/bradley-mannings-statement-taking-responsibility-for-releasing-documents-to-wikileaks, consulté le 30/03/15).

MONTAIGNE, M. de (2001 [1595]) : Les Essais, éd. par J. Céard, Paris, Éd. Le Livre de poche.

MONTESQUIEU (1995 [1717]) : Éloge de la sincérité, Paris, Éd. Mille et Une Nuits.

PAVEAU, M.-A. (2013) : Langage et morale. Une éthique des vertus discursives, Limoges, Lambert-Lucas.

PUTNAM, H. (2004 [2002]) : Fait/Valeur : la fin d'un dogme et autres essais, trad. par M. Caveribère et J.-P. Cometti, Paris/Tel-Aviv, Éd. de l'Éclat, p. 120.

STANFORD, B. (2000) : Don't Shoot the Messenger: How Our Growing Hatred of the Media Threatens Free Speech for All of Us, Rowman \& Littlefield.

\section{NOTES}

1. Dans un texte plus court, ou sur un support plus mobile comme un blog par exemple, j'aurais adopté l'écriture épicène et écrit cellui, puis locuteur.trice, messager.e, lanceur.euse d'alerte, etc., ainsi que diseur.se de vérité dans le titre. Ce choix militant est cependant fastidieux, contraignant et entame un peu la lisibilité des textes. Par ailleurs, dans la partie de cet article publiée en 2012, ne figurait pas cette écriture. J'y ai donc renoncé ici, mais le cœur y est et la vertu scripturale également.

2. Ce travail est fait dans l'ouvrage que j'ai consacré aux rapports entre langage et morale (Paveau, 2013).

3. Je donne, comme elle le demande, comme le respect relationnel minimum me semble le demander, et de manière militante également, son nom de femme à Chelsea Manning, qui effectue actuellement une transition difficile en prison.

4. Sur les notions de vertu discursive et d'ajustement à la vérité du monde, voir M.-A. Paveau (2013) et G. Labarta et S. Moirand ici même.

5. Voir le commentaire qu'en fait Nathan Fuller (2013) sur le site dédié à la défense de la libération de Chelsea Manning.

\section{RÉSUMÉS}

En linguistique de l'énonciation et en analyse du discours, l'énonciateur est rarement au centre de la description, les analyses restant largement logocentrées. Les diseurs de vérité sont des énonciateurs particulièrement intéressants car ils posent deux questions fondamentales à l'analyse du discours, à la philosophie morale, mais aussi à la société tout entière : la place de la parole dissensuelle dans le concert des discours sociaux, et le sort réservé à celui qui sort de l'ordre du discours. Après avoir décrit la manière dont les philosophes et le sens commun posent le problème de la vérité, l'article présente des figures de locuteurs de vérité, le messager, le parrèsiaste, le whistleblower et le lanceur d'alerte, à partir desquelles peut se poser la question de l'articulation entre éthique et linguistique. 
In enunciative linguistics and discourse analysis, the speaker is rarely the focus of the description, because linguistic analysis remain discourse centered. Truth tellers are particularly interesting speakers because they ask a core question to discourse analysis, moral philosophy, but also to the entire society: the role of dissensual speech in the stream of social discourse, and fate that comes out to those who break the order of discourse. After describing how philosophers and common sense problematize truth, the article presents figures of truth tellers, the messenger, the parresiast and the whistleblower, from which the question of the relationship between ethics and language can be examined.

\section{INDEX}

Mots-clés : éthique du discours, éthique énonciative, narcissisme moralisé, parrêsia, vérité, whistleblower

Keywords : discourse ethics, enunciative ethics, moralized narcissism, parresia, truth, whistleblower

\section{AUTEUR}

\section{MARIE-ANNE PAVEAU}

Pléiade (EA 7338), Université Paris 13 